\title{
Qualitative aspects of breathlessness in health and disease
}

\author{
J Smith, ${ }^{1}$ P Albert, ${ }^{2}$ E Bertella, ${ }^{3}$ J Lester, ${ }^{2}$ S Jack, ${ }^{2}$ P Calverley ${ }^{2}$
}

- Further details are published online only at http://thorax.bmj. com/content/vol64/issue8

${ }^{1}$ Respiratory Research Group, University of Manchester, University Hospital of South Manchester, Manchester, UK;

${ }^{2}$ School of Infection and Immunity, University of Liverpool, University Hospital Aintree, Liverpool, UK

${ }^{3}$ Cattedra di Malattie dell'apparato respiratprio, University of Brescia, Spedali Civili di Brescia, Italy

Correspondence to: Dr J A Smith, University of Manchester, ERC Building, Wythenshawe Hospital,

Manchester M23 9LT, UK jacky.smith@manchester.ac.uk

Received 28 August 2008 Accepted 30 March 2009 Published Online First 20 April 2009

\section{ABSTRACT}

Background: Patients with respiratory disease use many different expressions to describe the sensation they experience as breathlessness. Although previous analyses have identified multiple dimensions of breathlessness, there is little agreement about their number and nature. This study has applied a novel approach, principal component analysis (PCA), to understanding descriptions of breathlessness in health and disease and extracting representative components.

Methods: 202 patients (asthma $n=60$, chronic obstructive pulmonary disease $n=65$, interstitial lung disease $n=41$, idiopathic hyperventilation $n=36$ ) and 30 healthy volunteers were studied. All subjects performed spirometry and gave binary responses to 45 descriptions recalling their experience of breathlessness at the end of exercise; patients repeated this for resting breathlessness. PCA identified response patterns in the questionnaire data and extracted discriminatory components. Component scores were calculated for each individual using the regression method.

Results: PCA identified six distinct components of breathlessness on exercise, explaining $62.8 \%$ of the variance: (1) air hunger, (2) affective, (3) nociceptive, (4) regulation, (5) attention and (6) miscellaneous qualities. Rest components explaining $63.1 \%$ of variance were (1) affective, (2) air hunger, (3) nociceptive, (4) wheeze, (5) regulation and (6) miscellaneous. Components identified on exercise differed significantly between disease groups and controls and were related to percentage predicted forced vital capacity.

Conclusion: This analysis suggests that air hunger is the dominant sensation during exercise, while affective distress characterises resting breathlessness in patients with a range of respiratory disorders including idiopathic hyperventilation where lung mechanics are normal. This suggests that common mechanisms operate in qualitative aspects of breathlessness.

Breathlessness is one of the most frequent and distressing symptoms experienced by patients with lung disease and is usually defined as an uncomfortable awareness of breathing. ${ }^{1}$ Healthy subjects also experience breathlessness during exercise and this may be characterised as physiologically appropriate breathlessness. The clinical assessment of breathlessness usually focuses on the degree or intensity of the symptom, and much scientific effort has been dedicated to understanding the factors that determine the severity of breathlessness. ${ }^{2-4}$ Less attention has been paid to the quality of the sensation and whether this differs between conditions, although some believe this is the case..$^{5}$

Patients with a respiratory disease use a wide variety of terms to describe the sensations experienced when they become breathless. These sensations cannot be assessed objectively ${ }^{7}$ and, instead, representative verbal descriptors have been identified..$^{8}$ Although previous analyses in health ${ }^{9} 10$ and disease \& $^{11-13}$ have identified clusters of these descriptors (using cluster analysis), there is little consistency in their number and nature and, moreover, they are not sufficiently robust to aid in differential diagnosis.

An alternative approach to cluster analysis is to use principal component analysis (PCA) which uncovers the latent structure (dimensions) of a set of variables-in this case, breathlessness descriptors-by identifying important sources of variation. PCA has the advantage that it does not assume that distinct groups of descriptions exist; a variable can appear in two separate components and components can be allowed to correlate with each other. Differences in the interpretation of descriptions by subjects can therefore be accommodated while also avoiding the constraint of generating a hierarchic classification. This has been shown to be a useful technique for identifying patterns of respiratory symptoms in children ${ }^{14}$ but only one study has applied PCA to breathlessness, analysing a mixture of symptoms and qualitative descriptors in an attempt to identify subjects with medically unexplained breathlessness. ${ }^{15}$

We have applied PCA to the responses of healthy volunteers and discrete patient groups to descriptions of breathlessness. We chose our patient groups to represent a range of mechanical abnormalities, both fixed and variable, that are applied to the respiratory system, and our principal focus was on the recall of the sensation of breathlessness perceived at the end of exercise. We hypothesised that the quality of breathlessness would differ between healthy volunteers and patients with respiratory disease and would also differ between breathlessness recalled at end of exercise and that experienced at rest, although this analysis was confined to patients with disease where this might occur. Finally, we have explored the relationship between the components of breathlessness and spirometry as an objective measurement of altered lung mechanics.

\section{METHODS}

\section{Patients}

Consecutive patients attending the outpatient clinic and the respiratory function laboratory of University Hospital Aintree were recruited. Healthy control subjects of a similar age were identified from hospital staff and relatives of patients. 
Diagnoses were confirmed by review of the medical records and subjects categorised as chronic obstructive pulmonary disease (COPD), asthma, interstitial lung disease (ILD) and idiopathic hyperventilation (IHV) when they unequivocally met the established diagnostic criteria for these disorders ${ }^{16-18}$ (see online supplement for details). Patients with more than one condition causing breathlessness were excluded.

All patients performed spirometry using a wedge bellows spirometer (Vitalograph-R, Vitalograph Ltd, Buckinghamshire, UK); control subjects were tested using an ultrasonic portable spirometer (Easy One, NDD Medical Technologies, Zurich, Switzerland). The best of three manoeuvres has been reported, measured to ATS/ERS standards. ${ }^{19}$

\section{Questionnaire}

A questionnaire comprising 45 short phrases describing breathlessness and previously shown to be valid and reliable ${ }^{8}$ was completed by each subject twice. Patients were asked to think about how they felt when breathless at rest and to respond to all items (yes or no). Subjects were then asked to think about how they felt when breathless at end of exercise and to respond to the same set of items; healthy subjects completed only the exercise section. Unless they requested assistance, subjects were left alone and given as much time as they needed to complete the questionnaires.

\section{Data analysis}

All statistical analyses were performed using SPSS 13.0 (SPSS Inc, Chicago, Illinois, USA). PCA was used to identify response patterns in the questionnaire data both at rest and on exercise. ${ }^{20}$ PCA is an exploratory technique for investigating patterns within a set of variables - in the current analysis, responses to a series of descriptions of breathlessness. The large numbers of responses can be reduced to a much smaller number of representative components based on the covariance among responses. If subsets of symptoms are correlated, this suggests they are measuring aspects of a common underlying process; several components may suggest a series of underlying processes. The steps involved in each analysis were as follows.

\section{Item selection}

Redundant questions were removed (ie, question responses with partial correlation coefficients $>0.4$ to other question responses). To maximise the informative content of the analysis, items that correlated with several other variables were removed first.

\section{Component extraction}

PCA was used to generate the components and the numbers of components for analysis were selected based on the Eigenvalues and scree plots.

\section{Rotation}

Component rotation is used to improve the interpretability of the results. An oblique rotation was chosen (Promax), assuming that the components of breathlessness were unlikely to be entirely independent of one another. The loading of the items onto each component is a measure of the relationship between that item and the component-the greater the loading, the purer a measure of that component the item is. Only items with the conventional loading of 0.4 and above were interpreted.

\section{Component scores}

For each subject included in the analysis it is possible to calculate scores for the individual components. These scores are derived from the subjects' responses to the items comprising each component and their loadings. Scores were calculated using the regression method; for each component the scores were standardised; each has a mean value of 0 and a standard deviation of 1 . The scores indicate the relative importance of that qualitative component of breathlessness for each individual, not the intensity of breathlessness.

In addition, we applied agglomerative hierarchical cluster analysis (using the squared Euclidean distance as the dissimilarity measure) to the data set in order to compare the components with the clusters formed. This allowed comparison of our result with those previously generated using this questionnaire. $^{8}$

There are no available criteria against which the solution in a PCA can be tested. However, the solution can be assessed for face validity of the components and by examining the relationships between the component scores generated and other available variables. We therefore examined whether diagnostic grouping and also spirometric abnormality significantly predicted component scores using multivariate analysis of variance (MANOVA). If the predictor terms were significantly related to the component scores (according to the Pillai test), then individual associations between predictors and components were examined using specific tests. As patients with different conditions will inevitably have differences in spirometry, the relationships between component scores and spirometry were adjusted for differences due to diagnosis.

\section{RESULTS}

A total of 310 patients and 35 normal subjects were approached to fill in the questionnaire; 234 patients and 35 normal subjects agreed to take part in the study. Thirty-two patients and 5 healthy volunteers were excluded, leaving 202 patients and 30 normal subjects for analysis. The main reasons for exclusion were: inconsistency in answering the repeat questions ( $>1$ item answered differently) and failure to give a response to more than 5 questions. Patient characteristics, diagnoses and spirometric data are shown in table 1.

\section{Component extraction}

\section{Exercise}

For the analysis, data were pooled for the control and patient groups. Thirty-five items were included in the PCA (KaiserMeyer-Olkin measure of sampling adequacy 0.95, Bartlett's test of sphericity $<0.001$ ); item selection is summarised in table E1 in the online supplement. Six components with Eigenvalues $>1$ were identified (fig E1 in the online supplement), explaining $62.8 \%$ of the variance in the data. The main break in the scree plot was after one component as the first component explained the majority of the variance; however, the inclusion of the additional five components added a further $20 \%$ to the variance explained. The questionnaire items loading $>0.4$ onto each component are shown in table 2 , along with the proportion of variance explained by each component. We named each component based on the theme represented by the most strongly loading items. For the descriptions of breathlessness on exercise, the dominant component was air hunger (items referring to a need for more air). The other independent components explaining smaller percentages of the total variance were (in order of magnitude): affective; items suggesting 
Table 1 Characteristics of study subjects

\begin{tabular}{|c|c|c|c|c|c|}
\hline Diagnosis & Gender & $\begin{array}{l}\text { Mean age } \\
\text { (years) }\end{array}$ & $\mathrm{FEV}_{1} \%$ predicted & FVC $\%$ predicted & $\mathrm{FEV}_{1} / \mathrm{FVC}$ ratio \\
\hline Asthma $(n=60)$ & $19 \mathrm{M} / 41 \mathrm{~F}$ & $48.2(15.4)$ & $80.6(26.6)$ & $93.3(26.7)$ & $71.6(12.1)$ \\
\hline COPD $(n=65)$ & $32 \mathrm{M} / 33 \mathrm{~F}$ & $67.9(9.2)$ & $42.0(30.5-56.5)^{*}$ & $78.3(21.3)$ & $40.2(33.0-55.5)^{*}$ \\
\hline $\begin{array}{l}\text { Interstitial lung disease } \\
(\mathrm{n}=41)\end{array}$ & $24 \mathrm{M} / 17 \mathrm{~F}$ & $68.7(9.9)$ & $72.0(18.9)$ & $73.2(20.2)$ & $78.1(8.4)$ \\
\hline $\begin{array}{l}\text { Idiopathic } \\
\text { hyperventilation } \\
(\mathrm{n}=36)\end{array}$ & $8 \mathrm{M} / 28 \mathrm{~F}$ & $53.7(15.2)$ & $93.0(88.0-104.5)^{*}$ & $99.3(14.4)$ & $79.1(7.6)$ \\
\hline $\begin{array}{l}\text { Healthy controls } \\
(\mathrm{n}=30)\end{array}$ & $11 \mathrm{M} / 19 \mathrm{~F}$ & $53.8(12.7)$ & $106.5(16.0)$ & $113.3(17.2)$ & $77.2(6.3)$ \\
\hline
\end{tabular}

Data are mean (SD) except for ${ }^{*}$ median (interquartile range).

COPD, chronic obstructive pulmonary disease; $\mathrm{FEV}_{1}$, forced expiratory volume in $1 \mathrm{~s}$; FVC, forced vital capacity.

emotional distress, nociceptive; descriptions of unpleasant sensations, regulation; perceptions of inappropriately regulated breathing, attention; subjective awareness of breathing; and miscellaneous descriptions including sighing, air not tasting right and breath stopping. Several descriptions featured in more than one component (eg, air hunger loaded onto the air hunger and affective components).
Rest

Only data for the patients was used for this analysis. Thirtyfour items were included in the PCA (Kaiser-Meyer-Olkin measure of sampling adequacy 0.92 , Bartlett test of sphericity $<0.001)$; item selection is summarised in table E2 in the online supplement. Again, six components with Eigenvalues $>1$ were identified (fig E2 in the online supplement), explaining $63.1 \%$ of

Table 2 Pattern matrix: item loadings on exercise for patients and controls, six component solution with Promax rotation

\begin{tabular}{|c|c|c|c|c|c|c|}
\hline \multirow[b]{2}{*}{ Components ( $\%$ variance) items } & \multicolumn{6}{|c|}{ Component loadings } \\
\hline & 1 & 2 & 3 & 4 & 5 & 6 \\
\hline \multicolumn{7}{|l|}{ (1) Air hunger component (42.6\%) } \\
\hline Cannot breathe deeply enough & 0.86 & & & & & \\
\hline Need to take a deeper breath & 0.83 & & & & & \\
\hline Breathing too shallow & 0.77 & & & & & \\
\hline Not satisfied by my breathing & 0.67 & & & & & \\
\hline Can't get enough air into my chest & 0.67 & & & & & \\
\hline Cannot breathe enough & 0.67 & & & & & \\
\hline \multicolumn{7}{|l|}{ (2) Affective component (5.8\%) } \\
\hline Desperate for breath & & 0.89 & & & & \\
\hline Suffocating & & 0.86 & & & & \\
\hline Gasping for breath & & 0.74 & & & & \\
\hline Hunger for more air & 0.52 & 0.68 & & & & \\
\hline Fighting for breath & & 0.65 & & & & \\
\hline \multicolumn{7}{|l|}{ (3) Nociceptive component (4.6\%) } \\
\hline Chest aches & & & 0.82 & & & \\
\hline Chest feels tight & & & 0.77 & & & \\
\hline Raw sensation in chest & & & 0.74 & & & \\
\hline Wheezy & & & 0.65 & & & \\
\hline Raw sensation in throat & & & 0.50 & & & \\
\hline Winded in my chest & & & 0.41 & & & \\
\hline \multicolumn{7}{|l|}{ (4) Regulation component (3.7\%) } \\
\hline Breathing is too deep & & & & 0.88 & & \\
\hline Breathing is too fast & & & & 0.68 & & \\
\hline Breathing is too heavy & & & & 0.63 & & \\
\hline Breathing feels unpleasant & & & & 0.50 & & \\
\hline Can't control my breathing & & & & 0.41 & & \\
\hline \multicolumn{7}{|l|}{ (5) Attention component (3.1\%) } \\
\hline Puffed & & & & & 0.71 & \\
\hline Aware of my breathing & & & & & 0.66 & \\
\hline Need breath & 0.47 & & & & 0.53 & \\
\hline Short of breath & & & & & 0.52 & \\
\hline Out of breath & & & & & 0.49 & \\
\hline \multicolumn{7}{|l|}{ (6) Miscellaneous component (3.0\%) } \\
\hline Want to sigh & & & & & 0.42 & 0.61 \\
\hline Air does not taste right & & 0.48 & & & & 0.59 \\
\hline My breath stops & & 0.47 & & & & 0.55 \\
\hline
\end{tabular}

The magnitude of the component loadings represents how good each item is as an indicator of the component.

All items shown load $>0.40$ onto PCA components. 


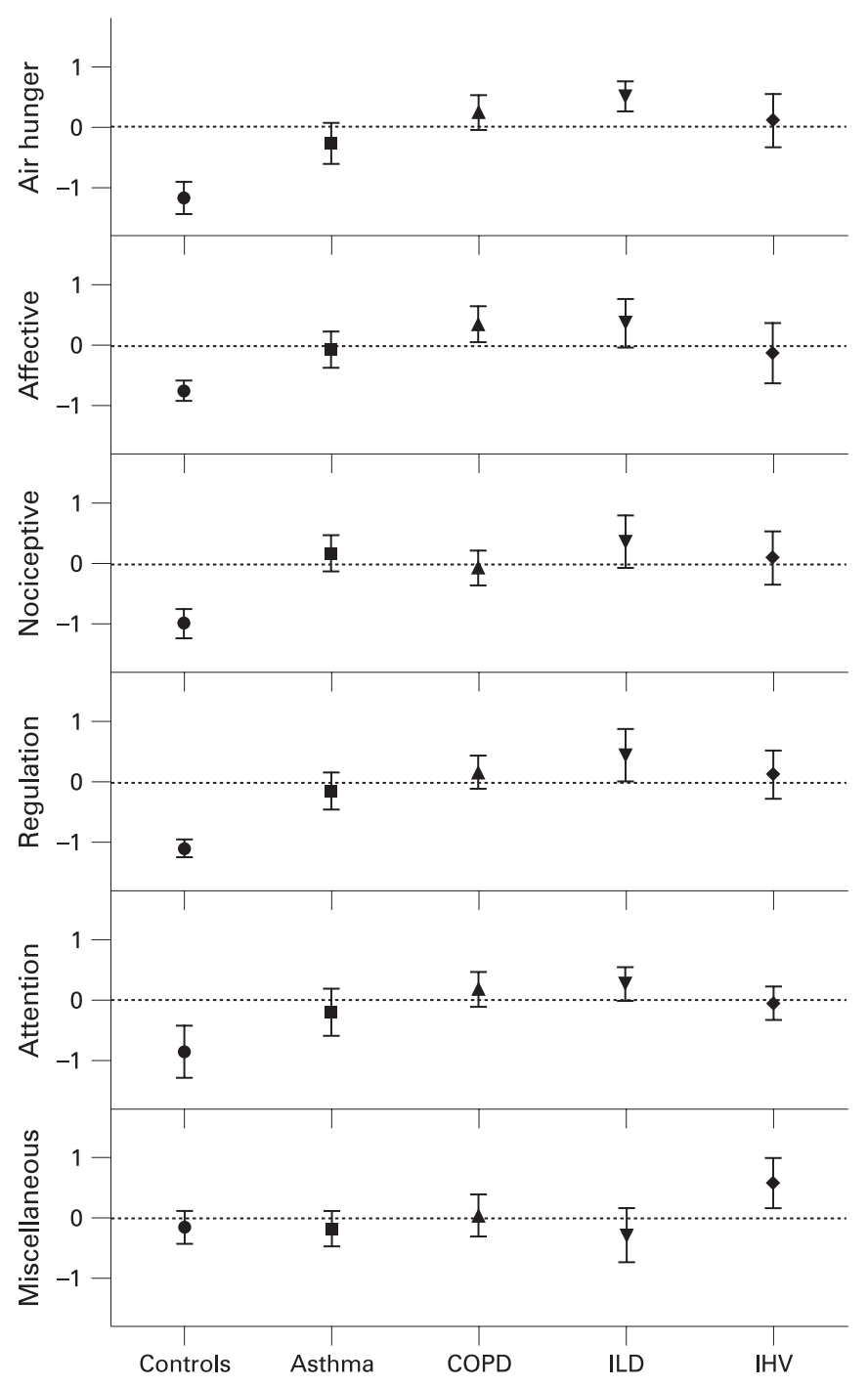

Figure 1 Comparison of diagnostic groups for each component score on exercise. Mean and 95\% confidence intervals are shown. COPD, chronic obstructive pulmonary disease; ILD, interstitial lung disease; IHV, idiopathic hyperventilation.

the variance in the descriptions. The questionnaire item loadings are shown in table E3 in the online supplement. The items in the components were either identical to or synonymous with those for exercise: (1) affective, (2) air hunger, (3) nociceptive, (4) wheeze, (5) regulation and (6) miscellaneous descriptions. An additional component representing wheeze emerged, but the attention component was not present. At rest the dominant component was the affective one.

To assess whether the absence of controls from the analysis had produced this change in the components and the variance explained, the PCA was repeated on the exercise data without the healthy volunteers. This confirmed that the air hunger component still explained most of the variance (see table E4 in online supplement) and the nociceptive items split into two components.

\section{Agglomerative hierarchical cluster analysis}

The exercise pooled control and patient data were analysed using all 45 descriptions. This generated a complex dendrogram. Using the criteria as previously applied by Elliott et a ${ }^{8}$ (squared Euclidean distance of 12.5), 17 clusters of descriptions were produced (fig E3 and table E5 in online supplement). At a Euclidean distance of 17.5 the cluster analysis gave six clusters. However, these did not seem to represent coherent themes-for example, the items in the air hunger and affective components appeared in a single cluster.

\section{Breathlessness component scores in health and disease}

Using the scores generated by the regression method, we were able to examine the validity of the components by assessing their ability to discriminate between control subjects and those with respiratory disease. Gender and age had no significant effect on any component score but, for the regulation component, there was a trend towards a significant gender difference at rest $(p=0.06)$ and on exercise $(p=0.09)$.

\section{Exercise component scores}

The diagnostic category significantly influenced the component score for all six exercise components (air hunger $p<0.001$, affective $p<0.001$, nociceptive $p<0.001$, regulation $p<0.001$, attention $p=0.001$ and miscellaneous $p=0.008)$. Post hoc analyses (Bonferroni correction for multiple comparisons) suggested that, compared with controls, the value of the component scores was greater for the air hunger component $(p<0.001$ for all diagnoses), the regulation component $(p<0.001$ for all diagnoses) and the nociceptive component ( $p<0.001$ for all diagnoses) (fig 1). There were also significantly different scores compared with controls for the affective component in asthma, COPD and ILD ( $p=0.005, p<0.001$ and $p<0.001$, respectively) but not IHV ( $p=0.125)$; a similar pattern occurred for the attentive component (COPD $p<0.001$, ILD $p=0.001$, and borderline significance for IHV $p=0.06$ and asthma $p=0.082$ ). In contrast, the miscellaneous component was significantly higher in IHV than asthma or ILD ( $p=0.03$ and $p=0.007$, respectively).

\section{Rest component scores}

The only significant difference between the diagnostic groups at rest was for the affective component $(p=0.04)$. Post hoc analysis suggested a higher score in subjects with COPD than in those with asthma ( $p=0.04$, fig E4 in online supplement).

\section{Breathlessness component scores and spirometry}

MANOVA models (exertion and rest) were generated with component scores as the dependant variables and spirometry as predictors. The ratio of forced expiratory volume in $1 \mathrm{~s}\left(\mathrm{FEV}_{1}\right)$ to forced vital capacity (FVC) was used to indicate airflow obstruction and FVC percentage predicted was used as a marker of volume change. All models were adjusted for age, gender and diagnosis.

In the multivariate model for exercise, FVC percentage predicted independently predicted all the breathlessness component scores except for the miscellaneous component (air hunger $p<0.001$, affective $p<0.001$, nociceptive $p=0.001$, regulation $p<0.001$ and attention $p=0.02$ ). Post hoc analyses suggested that FVC percentage predicted was significantly related to air hunger, affective and nociceptive components, independent of diagnosis (table 3 ). The $\mathrm{FEV}_{1} / \mathrm{FVC}$ ratio was not significant in the model $(p=0.37)$.

At rest, the $\mathrm{FEV}_{1} / \mathrm{FVC}$ ratio and FVC percentage predicted did not significantly influence the component scores when the model was adjusted for age, gender and diagnosis. 
Table 3 Post hoc analyses for variables significantly predicting breathlessness components on exercise in MANOVA

\begin{tabular}{lll}
\hline Components & Independent variables & p Values \\
\hline Exercise air hunger & FVC (\% predicted) Diagnosis & $0.02,<0.001$ \\
Exercise affective & FVC (\% predicted) & 0.004 \\
Exercise nociceptive & FVC (\% predicted) Diagnosis & $0.02,0.001$ \\
Exercise regulation & Diagnosis & $<0.001$ \\
Exercise attentive & Diagnosis & $<0.001$ \\
Exercise miscellaneous & - & - \\
\hline
\end{tabular}

Diagnosis reflects the independent impact of a specific diagnostic category.

FVC, forced vital capacity.

\section{DISCUSSION}

Although breathlessness is a cardinal symptom in respiratory disease, relatively little attention has been paid to its qualitative aspects. This reflects the difficulty in developing consistent themes from the range of attributes associated with this symptom. The replication of cluster analysis results in different patient groups has seldom been shown, while the resulting dendrograms are difficult to interpret. This is the first study to demonstrate that PCA can be successfully applied as an alternative to this approach and can identify different components of breathlessness experienced both at rest and end of exercise. The variation in the description of breathlessness at end of exercise was dominated by a single component relating to air hunger whereas, at rest, descriptions with emotional connotations (affective component) were the most important discriminators. Much smaller contributions were made by other components representing nociceptive sensations, attributes related to how breathing is regulated, wheeziness and the attention paid to the act of breathing. In general, the association of these variables and the diagnosis was closer on exercise than in the resting data. Gender and age had no significant effect on any component score, suggesting that the differences seen in perceived intensity of exertional breathlessness in older women ${ }^{21}$ are not associated with differences in quality of breathlessness.

To aid comparison with published data, we used the same list of descriptions of breathlessness as those reported by Elliott et al. ${ }^{8}$ Like them, we obtained a relatively complex dendrogram with a large number of clusters. This implies that breathlessness is a complicated sensation with a large number of dimensions, but we were not able to replicate the clusters formed and these differed significantly from the components indicated by the PCA. In contrast, PCA produced a smaller number of components with good face validity. When a simpler structure was derived from the cluster analysis (six clusters) it was apparent that the items in the air hunger and affective components from the PCA formed a single cluster (ie, were not discriminated by this technique). Furthermore, the remaining clusters lacked consistent themes.

Although the terms used to describe each cluster and each component are arbitrary and can be debated, the patients clearly identified qualities of unsatisfied inspiration within the grouping "air hunger" as the dominant perception of breathlessness on exercise. By contrast, terms related to emotional distress, "affective" attributes, were the ones most characteristic of breathlessness perceived at rest, reflecting the frightening nature of this sensation previously identified by patients such as those with COPD. ${ }^{22}$

The validity of the PCA was confirmed by our analysis of the derived component scores. We found that exercise components clearly distinguished between breathlessness in health and disease, but there were few significant differences between the different conditions for both rest and exercise. However, there was a difference in the affective component scores between asthma and COPD which could reflect the reported prevalence of depression in COPD, which is itself associated with chronic breathlessness. ${ }^{23} 24$

The dominance of air hunger as the major quality of breathlessness on exercise, irrespective of the differences in the mechanical behaviour of the lungs and those reporting it, is initially surprising. This may reflect a common mechanism generating this sensation on exercise. Air hunger is equally induced by both hypercapnia and hypoxia in health ${ }^{25}$ but is also experienced by ventilated quadriplegic subjects, ${ }^{26}$ supporting a model in which air hunger is mediated, at least in part, via the chemoreceptors and is independent of respiratory muscle contraction; this has been confirmed by studies in paralysed non-sedated volunteers. ${ }^{27}$ However, the adequacy of pulmonary inflation is also important in both inducing and relieving air hunger, ${ }^{28}$ suggesting that the sensation results from a balance between chemoreceptor and mechanoreceptor inputs. ${ }^{29}$ In disease, the factors modulating this interaction are complex. We speculate that mechanical limitation at end of exercise is a possible unifying explanation for the dominance of air hunger across our disease groups. Patients with significant airflow obstruction who report breathlessness show dynamic hyperinflation of their end-expiratory lung volume during exercise. ${ }^{30}$ Moreover, the degree of breathlessness increases significantly as the end-inspiratory lung volume approaches the inspiratory reserve volume. ${ }^{29} 31$ A similar situation may apply in ILD where the absolute inspiratory reserve volume is reduced. ${ }^{32}$ Although patients with IHV do not have any mechanical limitation to breathing at rest, they do show respiratory rather than cardiovascular limitation on exercise and the large tidal volume breathing they adopt at end of exercise is likely to encroach on the inspiratory reserve volume and generate a dissimilar sensation of unsatisfied inspiration. ${ }^{33}$ This interpretation is in keeping with our limited physiological data which showed that FVC rather than $\mathrm{FEV}_{1}$ was related to the sensation of breathlessness irrespective of the diagnosis. Thus, a common mechanism may explain why seemingly different diseases at rest produce qualitatively similar sensations.

Our study investigated respiratory diseases as a cause of breathlessness and omitted other conditions such as congestive heart failure where a mixture of cardiac and pulmonary abnormalities contribute directly or indirectly to dyspnoea. Our patient groups represent a spectrum of respiratory disease, all causing breathlessness but characterised by different mechanical abnormalities - the asthma and COPD groups representing variable and fixed airflow obstruction and the ILD group representing a purer form of elastic respiratory loading. Previous reports have not always included healthy controls in the same study as patients, ${ }^{5} 811$ and have not studied subjects with IHV. The subjects with IHV acted as a "positive control" group_subjects with symptoms of breathlessness but with normal spirometry, transfer factor and resting lung volumes. Patients with IHV are often felt to have psychological problems underlying their breathlessness, but our data indicate that the sensation experienced is the same as in patients with structural lung disease. The association of air hunger as the dominant exercise-related symptom was not different from that where breathlessness was due to organic factors, nor was there any stronger attribution of breathlessness to factors associated with the affective or emotional components we identified-something which might be expected if psychological factors played a dominant role in this condition. 
Our study has some limitations. We used a questionnaire developed by others to try and reduce variability between our datasets. However, the descriptions within this questionnaire are not strongly representative of the work or effort of breathing, an attribute previously described as being strongly related to breathlessness. ${ }^{34}$ However, our main interest was to identify the qualitative dimensions of breathlessness rather than dimensions inextricably related to the intensity of respiratory drive such as work and effort. It also would have been interesting to establish objectively the exercise capacity of all the participants, but PCA requires a large number of subjects making this impractical, notwithstanding the difficulties in identifying a suitable standardised test for all the disease groups studied. The range of spirometric abnormalities in the selected patient groups should be sufficient to encompass a wide range of exercise performance. Finally, we related breathlessness to a specific point during exercise-namely, maximum exercise performance-and it is possible that mechanisms operating earlier in exercise may be associated with a different quality of respiratory sensation. However, given the number of individuals questioned, identifying a specific point to consider made it easier for them to focus on the wide range of attributes they were asked to classify.

Our data suggest that the qualitative experience of breathlessness involves a variety of unpleasant sensations which are shared by a range of respiratory conditions irrespective of their aetiology. Whether the same is true for other conditions where breathlessness limits exercise remains to be determined. We have not identified significant differences in the qualitative attributes of breathlessness which are disease-related, so the present clinical practice of quantifying the intensity of the sensation relative to the task which produces it appears to capture the important attributes of breathlessness.

Funding: British Lung Foundation.

Competing interests: None.

Ethics approval: The study was approved by the local research ethics committee and written consent was obtained from all participants.

\section{REFERENCES}

1. Burki NK. Dyspnea. Lung 1987;165:269-77.

2. Mahler DA, Weinberg DH, Wells CK, et al. The measurement of dyspnea. Contents, interobserver agreement, and physiologic correlates of two new clinical indexes. Chest 1984:85:751-8.

3. Killian KJ. Assessment of dyspnoea. Eur Respir J 1988;1:195-7.

4. Cockcroft A, Adams L, Guz A. Assessment of breathlessness. $0 \mathrm{~J}$ Med 1989:72:669-76.

5. Simon PM, Schwartzstein RM, Weiss JW, et al. Distinguishable types of dyspnea in patients with shortness of breath. Am Rev Respir Dis 1990:142:1009-14.

6. Scano G, Stendardi L, Grazzini M. Understanding dyspnoea by its language. Eur Respir J 2005:25:380-5.

7. Schwartzstein RM, Cristiano LM. Qualities of respiratory sensation. In: Adams L, Guz A, eds. Lung biology in health and disease, Vol 90: Respiratory sensation. New York: Marcel Dekker, 1996:125-50.
8. Elliott MW, Adams L, Cockcroft A, et al. The language of breathlessness. Use of verbal descriptors by patients with cardiopulmonary disease. Am Rev Respir Dis 1991;144:826-32.

9. Simon PM, Schwartzstein RM, Weiss JW, et al. Distinguishable sensations of breathlessness induced in normal volunteers. Am Rev Respir Dis 1989;140:1021-7.

10. Harver A, Mahler DA, Schwartzstein RM, et al. Descriptors of breathlessness in healthy individuals: distinct and separable constructs. Chest 2000;118:679-90.

11. Wilcock A, Crosby V, Hughes A, et al. Descriptors of breathlessness in patients with cancer and other cardiorespiratory diseases. J Pain Symptom Manage 2002;23:182-9.

12. von Leupoldt A, Balewski S, Petersen $S$, et al. Verbal descriptors of dyspnea in patients with COPD at different intensity levels of dyspnea. Chest 2007;132:141-7.

13. Moy ML, Lantin ML, Harver A, et al. Language of dyspnea in assessment of patients with acute asthma treated with nebulized albuterol. Am J Respir Crit Care Med 1998; 158:749-53.

14. Smith JA, Drake R, Simpson A, et al. Dimensions of respiratory symptoms in preschool children: population-based birth cohort study. Am J Respir Crit Care Med 2008;177:1358-63.

15. Han J, Zhu Y, Li S, et al. The language of medically unexplained dyspnea. Chest 2008;133:961-8.

16. American Thoracic Society. Idiopathic pulmonary fibrosis: diagnosis and treatment. International consensus statement. American Thoracic Society (ATS), and the European Respiratory Society (ERS). Am J Respir Crit Care Med 2000;161 (2 Pt 1):646-64.

17. Celli BR, MacNee W. Standards for the diagnosis and treatment of patients with COPD: a summary of the ATS/ERS position paper. Eur Respir J 2004;23:932-46.

18. Global Initiative for Asthma (GINA). Global strategy for asthma management and prevention. GINA, 2002 (updated 2005).

19. Miller MR, Hankinson J, Brusasco V, et al. Standardisation of spirometry. Eur Respir J 2005:26:319-38.

20. Tabachnick BG, Fidell LS, eds. Principal components and factor analysis. In: Using multivariate statistics. 5th ed. Boston: Allyn and Bacon, 2007:607-75

21. Ofir D, Laveneziana $P$, Webb KA, et al. Sex differences in the perceived intensity of breathlessness during exercise with advancing age. J Appl Physiol 2008;104:1583-93.

22. Rennard S, Decramer M, Calverley PM, et al. Impact of COPD in North America and Europe in 2000: subjects' perspective of Confronting COPD International Survey. Eur Respir J 2002;20:799-805.

23. Laurin C, Lavoie KL, Bacon SL, et al. Sex differences in the prevalence of psychiatric disorders and psychological distress in patients with COPD. Chest 2007;132:148-55.

24. Yohannes AM, Baldwin RC, Connolly MJ. Depression and anxiety in elderly outpatients with chronic obstructive pulmonary disease: prevalence, and validation of the BASDEC screening questionnaire. Int J Geriatr Psychiatry 2000;15:1090-6.

25. Moosavi SH, Golestanian E, Binks AP, et al. Hypoxic and hypercapnic drives to breathe generate equivalent levels of air hunger in humans. J Appl Physiol 2003;94:141-54.

26. Banzett RB, Lansing RW, Reid MB, et al. 'Air hunger' arising from increased $\mathrm{PCO}_{2}$ in mechanically ventilated quadriplegics. Respir Physiol 1989;76:53-67.

27. Banzett RB, Lansing RW, Brown R, et al. 'Air hunger' from increased $\mathrm{PCO}_{2}$ persists after complete neuromuscular block in humans. Respir Physiol 1990;81:1-17.

28. Manning HL, Shea SA, Schwartzstein RM, et al. Reduced tidal volume increases 'air hunger' at fixed $\mathrm{PCO}_{2}$ in ventilated quadriplegics. Respir Physiol 1992;90:19-30.

29. O'Donnell DE, Banzett RB, Carrieri-Kohlman V, et al. Pathophysiology of dyspnea in chronic obstructive pulmonary disease: a roundtable. Proc Am Thorac Soc 2007:4:145-68.

30. O'Donnell DE, Lam M, Webb KA. Measurement of symptoms, lung hyperinflation, and endurance during exercise in chronic obstructive pulmonary disease. Am J Respir Crit Care Med 1998;158(5 Pt 1):1557-65.

31. O'Donnell DE, Voduc N, Fitzpatrick M, et al. Effect of salmeterol on the ventilatory response to exercise in chronic obstructive pulmonary disease. Eur Respir $J$ 2004;24:86-94.

32. O'Donnell DE, Chau LK, Webb KA. Qualitative aspects of exertional dyspnea in patients with interstitial lung disease. J Appl Physiol 1998;84:2000-9.

33. Jack S, Rossiter HB, Pearson MG, et al. Ventilatory responses to inhaled carbon dioxide, hypoxia, and exercise in idiopathic hyperventilation. Am J Respir Crit Care Med 2004;170:118-25.

34. O'Donnell DE, Bertley JC, Chau LK, et al. Qualitative aspects of exertional breathlessness in chronic airflow limitation: pathophysiologic mechanisms. Am J Respir Crit Care Med 1997:155:109-15. 